\title{
Correction to: A primer on nerve agents: what the emergency responder, anesthesiologist, and intensivist needs to know
}

\author{
Keith Candiotti, MD
}

Published online: 21 November 2017

(c) Canadian Anesthesiologists' Society 2017

\section{Correction to: Can J Anesth/J Can Anesth (2017) 64:1059-1070 \\ DOI 10.1007/s12630-017-0920-2}

In the article entitled: "A primer on nerve agents: what the emergency responder, anesthesiologist, and intensivist needs to know" published in the October 2017 issue of the Journal, Can J Anesth 2017; 64: 1059-1070, two doses in Table 3 contained errors and have now been corrected (and highlighted in bold) in the revised Table 3. In the table on page 1064, next to "Pralidoxime/Obidoxime/HI6", the second column should read: "Pralidoxime- Mild cases: 1-2 g $i v$ over 5-10 min or im". Also in the same row, the third column should read: "Individual doses should not exceed 2 g”.

The online version of the original article can be found under https:// doi.org/10.1007/s12630-017-0920-2.

K. Candiotti, MD

University of Miami, Miller School of Medicine, Miami, FL, USA

K. Candiotti, MD ( $\bowtie)$

Department of Anesthesiology, Perioperative Medicine and Pain Management, Jackson Memorial Hospital, (C-302), University of Miami, 1611 NW 12th Ave, Miami, FL 33136, USA

e-mail: kcandiotti@miami.edu 
Table 3 Antidotal treatment of nerve agent-exposed patients (adapted from reference) ${ }^{31}$

\begin{tabular}{|c|c|c|}
\hline Drug & Dosage & Additional information \\
\hline Atropine & $\begin{array}{l}\text {-Mild case: } 2 \mathrm{mg} i v \text { repeated every } 20 \mathrm{~min} \text { until full } \\
\text { atropinization } \\
\text { Children: } 0.02 \mathrm{mg} \cdot \mathrm{kg}^{-1} \\
\text {-Moderate/Severe exposure: } 2 \mathrm{mg} i v \text { repeated every } 5-10 \mathrm{~min} \\
\quad \text { until full atropinization } \\
\text { Children: } 2 \mathrm{mg} \text { or } 0.02-0.08 \mathrm{mg} \cdot \mathrm{kg}^{-1}\end{array}$ & $\begin{array}{l}\text {-In elderly patients, after initial } 2 \text {-mg dose, consider } \\
\text { decreased repeat doses of } 1 \mathrm{mg} \\
\text {-MARK } 1 \text { autoinjector is a } 2-\mathrm{mg} \mathrm{im} \text { dose } \\
\text {-In severe intoxication, } 6 \mathrm{mg} \text { may be needed in first hour, } \\
10-20 \mathrm{mg} \text { in the first } 2-3 \mathrm{hr} \text {, and } 50-100 \mathrm{mg} \text { over } 24 \mathrm{hr} \text { in } \\
\text { severe cases } \\
\text {-Glycopyrrolate may be useful to treat peripheral } \\
\text { symptoms. It does not cross to the CNS }\end{array}$ \\
\hline Scopolamine & $\begin{array}{l}\text {-Mild cases: } 0.25 \mathrm{mg} \text { im every } 4-6 \mathrm{hr} \\
\text {-Moderate/Severe exposure: } 0.25 \mathrm{mg} i v \text { repeated every } 30 \mathrm{~min} \\
\text { for } 2 \text { doses. Then } q 4-6 \mathrm{hr} \text { as needed }\end{array}$ & -Do not use in children \\
\hline $\begin{array}{l}\text { Pralidoxime/ } \\
\text { Obidoxime/ } \\
\text { HI-6 }\end{array}$ & $\begin{array}{l}\text { Pralidoxime-Mild cases: } \mathbf{1 - 2} \text { g } \mathbf{i v} \text {, over } \mathbf{5 - 1 0} \text { min or } \text { im } \\
\text { Children: } 15-25 \mathrm{mg} \cdot \mathrm{kg}^{-1} \mathrm{iv} \text { or } \mathrm{im} \\
\text { Infants: } 15 \mathrm{mg} \cdot \mathrm{kg}^{-1} \mathrm{iv} \\
\text {-Moderate/Severe cases: Same dose but intravenously preferred } \\
\text { Obidoxime-Mild cases: } 250 \mathrm{mg} \text { im every } 2 \mathrm{hr} \text { to a maximum of } 3 \\
\text { doses } \\
\text {-Children }<2 \mathrm{yr} \text { : } 62.5 \mathrm{mg} \text { im every } 2 \mathrm{hr} \text { to a maximum of } 3 \\
\text { doses. } 2-10 \mathrm{yr}: 125 \mathrm{mg} \text { every } 2 \mathrm{hr} \text { to a maximum of } 3 \text { doses. } \\
\text { Over } 10 \mathrm{yr} \text { as an adult } \\
\text {-Moderate/Severe exposure: } 250 \mathrm{mg} i v \text { over } 30 \text { min, maximum } 3 \\
\text { doses typically but up to } 2 \mathrm{~g} \text { if clinically effective } \\
\text {-Children: } 250 \mathrm{mg} \text { every } 2 \mathrm{hr} \text { (maximum of } 3 \text { doses but an } \\
\text { additional } 5 \text { doses may be given if proving effective) } \\
\text { HI-6: Autoinjector dose is } 500 \mathrm{mg} \text {, typically a single dose may } \\
\text { prove efficacious in mild poisoning. Higher dosing may be } \\
\text { required in more significant exposure. ( } 3 \text { doses) }\end{array}$ & $\begin{array}{l}\text {-MARK } 1 \text { autoinjector is a } 600 \text {-mg im dose } \\
\text {-Individual doses should not exceed } \mathbf{2} \mathbf{~ g} \\
\text {-If given intravenously should be given slowly }\end{array}$ \\
\hline Benzodiazepines & $\begin{array}{l}\text {-Diazepam: } 0.2 \mathrm{mg} \cdot \mathrm{kg}^{-1} \text { or } 2-10 \mathrm{mg} i v \text { in adults } \\
\text { Children } 0.2-0.4 \mathrm{mg} \cdot \mathrm{kg}^{-1} \\
\text {-Midazolam } 0.1-0.2 \mathrm{mg} \cdot \mathrm{kg}^{-1} \mathrm{im} \text { or } i v\end{array}$ & $\begin{array}{l}\text {-Diazepam autoinjector is a } 10-\mathrm{mg} \text { im dose } \\
\text {-Individual diazepam doses should not exceed } 10 \mathrm{mg} \\
\text {-Total doses of diazepam to suppress seizures in adults may } \\
\text { be as high as } 30-40 \mathrm{mg}\end{array}$ \\
\hline
\end{tabular}

All drug doses are estimates. In cases of severe intoxication, especially with organophosphate pesticides, additional doses may be required above the stated maximum doses. CNS = central nervous system 